STRUCTURAL BIOLOGY COMMUNICATIONS

ISSN 2053-230X

Received 22 October 2015

Accepted 21 December 2015

Edited by W. N. Hunter, University of Dundee, Scotland

Keywords: FACT; Spt16; histone chaperone; histones; pita-bread fold; aminopeptidase.

PDB reference: $N$-terminal domain of human Spt16, 5e5b

Supporting information: this article has supporting information at journals.iucr.org/f

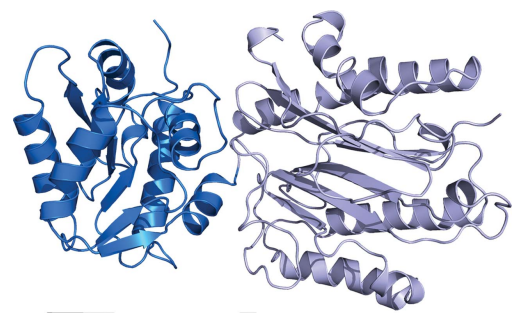

OPEN $\odot$ ACCESS

\section{Structure of the human histone chaperone FACT Spt16 N-terminal domain}

\author{
G. Marcianò and D. T. Huang* \\ Cancer Research UK Beatson Institute, Garscube Estate, Switchback Road, Glasgow G61 1BD, Scotland. \\ *Correspondence e-mail: d.huang@beatson.gla.ac.uk
}

The histone chaperone FACT plays an important role in facilitating nucleosome assembly and disassembly during transcription. FACT is a heterodimeric complex consisting of Spt16 and SSRP1. The N-terminal domain of Spt16 resembles an inactive aminopeptidase. How this domain contributes to the histone chaperone activity of FACT remains elusive. Here, the crystal structure of the N-terminal domain (NTD) of human Spt16 is reported at a resolution of $1.84 \AA$. The structure adopts an aminopeptidase-like fold similar to those of the Saccharomyces cerevisiae and Schizosaccharomyces pombe Spt16 NTDs. Isothermal titration calorimetry analyses show that human Spt16 NTD binds histones H3/H4 with low-micromolar affinity, suggesting that Spt16 NTD may contribute to histone binding in the FACT complex. Surface-residue conservation and electrostatic analysis reveal a conserved acidic patch that may be involved in histone binding.

\section{Introduction}

Eukaryotes package their DNA into nucleosomes, which consist of four histone proteins, $\mathrm{H} 2 \mathrm{~A}, \mathrm{H} 2 \mathrm{~B}, \mathrm{H} 3$ and $\mathrm{H} 4$, that associate to form two $\mathrm{H} 2 \mathrm{~A} / \mathrm{H} 2 \mathrm{~B}$ heterodimers and one $\mathrm{H} 3 / \mathrm{H} 4$ heterotetramer. Two $\mathrm{H} 2 \mathrm{~A} / \mathrm{H} 2 \mathrm{~B}$ heterodimers and one $\mathrm{H} 3 / \mathrm{H} 4$ heterotetramer associate to form an octamer around which $147 \mathrm{bp}$ of DNA are wrapped (Luger et al., 1997). Histone chaperones play important roles in directing histone deposition into DNA and histone disassembly from the nucleosome to facilitate DNA replication, transcription and DNA-damage response (Gurard-Levin et al., 2014).

Facilitating chromatin transcription (FACT) is a histone chaperone consisting of a heterocomplex of suppressor of Ty 16 (Spt16) and structure-specific recognition protein 1 (SSRP1). Early in vitro studies showed that FACT displaces the $\mathrm{H} 2 \mathrm{~A} / \mathrm{H} 2 \mathrm{~B}$ dimer from the nucleosome template to facilitate RNA polymerase II-driven transcription and has the ability to deposit histones onto DNA (Orphanides et al., 1998, 1999). Subsequent studies showed that when FACT is incubated with $\mathrm{H} 2 \mathrm{AX}$ and the nucleosome, it can catalyse the exchange of $\mathrm{H} 2 \mathrm{AX}$ with $\mathrm{H} 2 \mathrm{~A}$ within the nucleosome (Heo et al., 2008) and compete against DNA for $\mathrm{H} 2 \mathrm{~A} / \mathrm{H} 2 \mathrm{~B}$ binding but not the H3/H4 tetramer (Winkler et al., 2011). These studies support a model in which FACT functions as an $\mathrm{H} 2 \mathrm{~A} / \mathrm{H} 2 \mathrm{~B}$ exchanger. A recent study has shown that yeast FACT can relax DNA-histone interactions without removing $\mathrm{H} 2 \mathrm{~A} / \mathrm{H} 2 \mathrm{~B}$ and the loss of dimer is likely to be owing to reorganization of the destabilized nucleosome (Xin et al., 2009). Nonetheless, to destabilize the nucleosome, FACT is likely to disrupt DNAhistone interactions by binding directly to the histones. Indeed, early studies showed that FACT and both subunits 
of FACT can bind histones (Belotserkovskaya et al., 2003; Formosa et al., 2001). Moreover, affinity analyses showed that FACT interacts with the core domains and $\mathrm{N}$-terminal tails of both histones $\mathrm{H} 2 \mathrm{~A} / \mathrm{H} 2 \mathrm{~B}$ and $\mathrm{H} 3 / \mathrm{H} 4$ through several synergistic binding events involving both Spt16 and SSRP1 (Winkler et al., 2011). How Spt16 and SSRP1 interact with histones remains elusive.

SSRP1 contains an N-terminal Spt16-binding domain followed by a middle domain (MD) and a C-terminal HMG domain. SSRP1 MD adopts two pleckstrin-homology (PH) folds and has been shown to bind histone $\mathrm{H} 3 / \mathrm{H} 4$ (VanDemark et al., 2006; Zunder et al., 2012). Genetic analysis of yeast SSRP1 MD revealed that some mutations, including Q308K, can induce temperature sensitivity, hydroxyurea sensitivity and an Spt- phenotype, suggesting the importance of this domain in transcription and DNA replication, possibly through interactions with histones (VanDemark et al., 2006). The HMG domain has been shown to bind nucleosomal DNA and this interaction causes DNA bending, leading to minorgroove widening that may disrupt DNA-histone interactions (Masse et al., 2002; Kasai et al., 2005).

Spt16 consists of an NTD followed by an SSRP1 dimerization domain, MD and C-terminal domain. Spt16 MDs from Chaetomium thermophilum and Saccharomyces cerevisiae have been shown to interact with histone $\mathrm{H} 3 / \mathrm{H} 4$, and C. thermophilum Spt16 MD also binds H2/H2B (Kemble et al., 2013; Hondele et al., 2013). The structure of Spt16 MD reveals two PH domains in tandem and it shares structural similarity with SSRP1 MD and RTT106; however, it has three additional $\alpha$-helices located at the $\mathrm{C}$-terminus that form a U-turn motif. This motif interacts with the $\mathrm{H} 2 \mathrm{~A} / \mathrm{H} 2 \mathrm{~B}$ dimer via hydrophobic interactions with residues on the $\mathrm{H} 2 \mathrm{~B} \alpha 1$ helix (Hondele et al., 2013). Interestingly, most of the Spt16 mutations that affect FACT activity are in Spt16 MD (Myers et al., 2011; Formosa et al. 2001). These mutations can be suppressed by destabilizing $\mathrm{H} 2 \mathrm{~A} / \mathrm{H} 2 \mathrm{~B}$ dimer and $\mathrm{H} 3 / \mathrm{H} 4$ tetramer interactions (Myers et al., 2011; McCullough et al., 2011; Hainer et al., 2012), suggesting that Spt16 MD is responsible for establishing important interactions with the nucleosome. The Spt16 C-terminal domain consists of highly intrinsically disordered acidic residues. Removal of these regions hampers the ability of FACT to bind nucleosomes and histones and to promote transcription (Belotserkovskaya et al., 2003; Myers et al., 2011). A recent structural study showed that the Spt16 and SSRP1 C-terminal flexible acidic residues contain a tyrosine or phenylalanine residue, respectively, that binds to a hydrophobic pocket in H2B (Kemble et al., 2015). This interaction overlaps with nucleosomal DNA-H2A/H2B interactions, suggesting that FACT might promote reorganization of the nucleosomes by competing with DNA for histone binding.

The function of Spt16 NTD remains unclear. Studies in yeast showed that deletion of this domain or mutations in specific conserved residues do not disrupt essential functions of yeast FACT. However, when combined with the yeast SSRP1 MD Q308K mutation that causes defects in DNA replication and transcription, the effect is lethal, suggesting that Spt16 NTD functionally overlaps with SSRP1 MD
Table 1

Crystallization.

\begin{tabular}{|c|c|}
\hline Method & Hanging-drop vapour diffusion \\
\hline Plate type & 24-well hanging-drop plate \\
\hline Temperature (K) & 292 \\
\hline Protein concentration $\left(\mathrm{mg} \mathrm{ml}^{-1}\right)$ & 13.3 \\
\hline $\begin{array}{l}\text { Buffer composition of protein } \\
\text { solution }\end{array}$ & $\begin{array}{l}25 \mathrm{~m} M \text { Tris- } \mathrm{HCl} \text { pH } 7.6,150 \mathrm{~m} M \mathrm{NaCl}, \\
1 \mathrm{~m} M \text { DTT }\end{array}$ \\
\hline Composition of reservoir solution & $\begin{array}{l}0.1 M \text { sodium acetate } \mathrm{pH} 5.0,17 \%(w / v) \\
\text { PEG } 3350,0.1 M \text { ammonium iodide }\end{array}$ \\
\hline Volume and $\mathrm{r}$ & $2 \mu \mathrm{l}, 1: 1$ ratio \\
\hline Volume of reservoir $(\mu \mathrm{l})$ & 0.5 \\
\hline
\end{tabular}

Table 2

Data collection and processing.

Values in parentheses are for the outer shell.

\begin{tabular}{ll}
\hline Diffraction source & DLS beamline I02 \\
Wavelength $(\AA)$ & 0.979493 \\
Temperature $(\mathrm{K})$ & 100 \\
Detector & Pilatus $6 \mathrm{M}$ \\
Crystal-to-detector distance $(\mathrm{mm})$ & 264.6 \\
Rotation range per image $\left({ }^{\circ}\right)$ & 0.85 \\
Total rotation range $\left(^{\circ}\right)$ & 90 \\
Exposure time per image $(\mathrm{s})$ & 0.5 \\
Space group & $F 432$ \\
$a, b, c(\AA)$ & $246.57,246.57,246.57$ \\
$\alpha, \beta, \gamma\left({ }^{\circ}\right)$ & $90,90,90$ \\
Mosaicity $\left({ }^{\circ}\right)$ & 0.13 \\
Resolution range $(\AA)$ & $87.18-1.84(1.88-1.84)$ \\
Total No. of reflections & $1124024(72859)$ \\
No. of unique reflections & $56315(4094)$ \\
Completeness $(\%)$ & $100.0(100.0)$ \\
Multiplicity & $20.0(17.8)$ \\
$\langle I / \sigma(I)\rangle$ & $28.1(4.2)$ \\
$R_{\text {r.i.m }}{ }^{\dagger}$ & $0.084(0.822)$ \\
$R_{\text {p.i.m. }}$ & $0.019(0.193)$ \\
Overall $B$ factor from Wilson plot $\left(\AA^{2}\right)$ & 22.67 \\
\hline
\end{tabular}

$\dagger$ Estimated $R_{\text {r.i.m. }}=R_{\text {merge }}[N /(N-1)]^{1 / 2}$, where $N$ is the data multiplicity.

(O’Donnell et al., 2004; VanDemark et al., 2008). Furthermore, genetic analyses showed that Spt16 NTD might interact with the C-terminal extension of H2A (VanDemark et al., 2008). The structures of Spt16 NTD from S. cerevisiae and Schizosaccharomyces pombe (PDB entries 3bip and 3cb5, respectively) reveal an aminopeptidase-like domain that resembles a 'pita-bread' fold and which has lost its aminopeptidase activity (Stuwe et al., 2008; VanDemark et al., 2008). Studies on $S$. pombe Spt16 NTD showed that it forms a tight complex with histone $\mathrm{H} 3 / \mathrm{H} 4$. Moreover, it binds $\mathrm{H} 3$ and $\mathrm{H} 4 \mathrm{~N}$-terminal tails with low-micromolar affinity (Stuwe et al., 2008). In contrast, no binding of histone N-terminal tails could be observed with $S$. cerevisiae Spt16 NTD (VanDemark et al., 2008). To further elucidate the role of Spt16 NTD in histone interactions, we determined the crystal structure of human Spt16 NTD and examined its histone-binding ability.

\section{Materials and methods}

\subsection{Macromolecule production}

The gene for human Spt16 NTD (residues 1-510) was cloned into pGEX-4T-1 vector (Novagen) using standard PCR ligation techniques such that the protein is expressed with an 
N-terminal glutathione $S$-transferase (GST) tag followed by a TEV protease cleavage site. The resulting vector was transformed into Escherichia coli BL21 (DE3) Gold competent cells. The cells were grown at $310 \mathrm{~K}$ in $\mathrm{LB}$ to an $\mathrm{OD}_{600}$ of $\sim 0.7-0.8$ and then induced with $0.2 \mathrm{~m} M$ isopropyl $\beta$-D-1- thiogalactopyranoside overnight at $293 \mathrm{~K}$. The following day, the cells were harvested and resuspended in $50 \mathrm{~m} M$ Tris- $\mathrm{HCl}$ pH 7.6, $0.2 M \mathrm{NaCl}, 1 \mathrm{~m} M$ DTT, $2.5 \mathrm{~m} M$ phenylmethylsulfonyl fluoride, $1 \mathrm{mg} \mathrm{ml}^{-1}$ lysozyme and then subjected to a freezethaw cycle. The cells were lysed by sonication and then

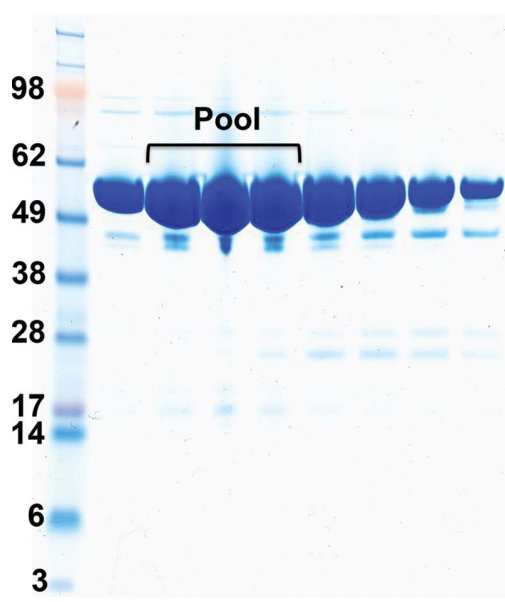

(a)

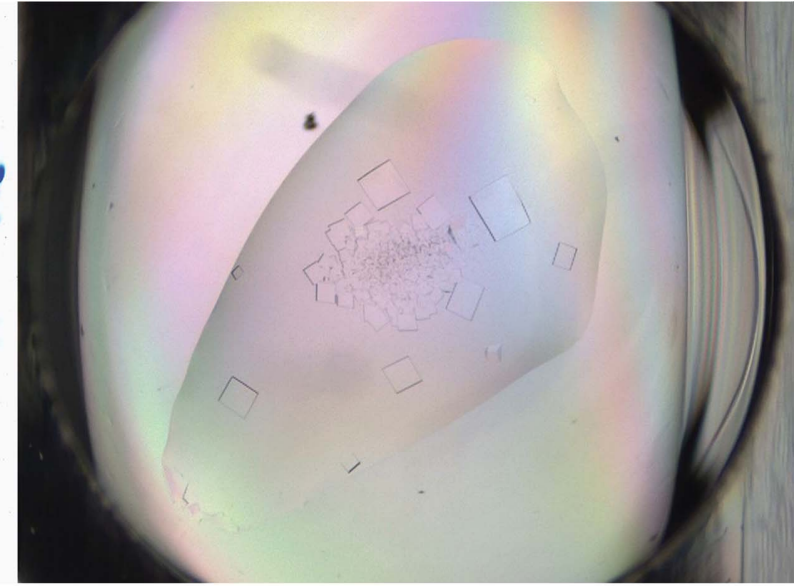

(b)

Figure 1

Purity and crystals of human Spt16 NTD. (a) SDS-PAGE showing fractions of human Spt16 NTD after Superdex 75 gel-filtration chromatography. (b) Crystals of human Spt16 NTD.

(1-175)

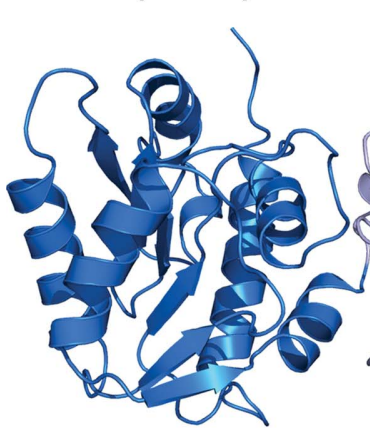

N-lobe

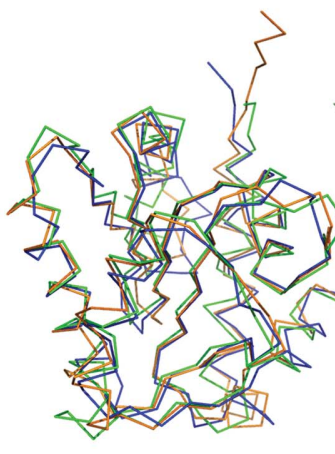

Figure 2 175, blue) and a C-terminal lobe (residues 176-432, light blue). $(c, d)$ Superimposition of human (blue), S. cerevisiae (green; PDB entry 3 bip) and $S$. pombe (orange; PDB entry 3cb5) Spt16 NTD structures. $(c)$ and $(d)$ are displayed in the same orientations as $(a)$ and $(b)$, respectively. Arrows indicate variations in the loops owing to differences in protein sequence and length. 
centrifuged at $48000 \mathrm{~g}$ for $25 \mathrm{~min}$ at $277 \mathrm{~K}$ using a JA-25.50 rotor (Beckman Coulter) to remove cell debris. GST-Spt16 NTD was purified from the lysate by glutathione Sepharose affinity chromatography. The GST tag was released by incubation with TEV protease and subsequently removed with a glutathione Sepharose column. Cleaved Spt16 NTD was then purified by anion-exchange chromatography (Source Q, GE Life Sciences) in buffer consisting of $50 \mathrm{~m} M$ Tris- $\mathrm{HCl} \mathrm{pH}$ 7.6, $1 \mathrm{~m} M$ DTT, $0-1 M \mathrm{NaCl}$ and by size-exclusion chromatography (Superdex 75, GE Life Sciences) in buffer consisting of $25 \mathrm{~m} M$ Tris- $\mathrm{HCl} \mathrm{pH}$ 7.6, $150 \mathrm{~m} M \mathrm{NaCl}, 1 \mathrm{~m} M$ DTT. Fractions containing Spt16 NTD were pooled together and concentrated to about $13.3 \mathrm{mg} \mathrm{ml}^{-1}$. The protein was then cooled in liquid nitrogen and stored at $193 \mathrm{~K}$. Gallus gallus histone octamer proteins ( $\mathrm{H} 2 \mathrm{~A}, \mathrm{H} 2 \mathrm{~B}, \mathrm{H} 3$ and $\mathrm{H} 4)$ were purified from chicken blood (Lambert et al., 1999) and separated into histones H2A/ $\mathrm{H} 2 \mathrm{~B}$ and $\mathrm{H} 3 / \mathrm{H} 4$ by cation-exchange chromatography (Source $\mathrm{S}$, GE Life Sciences) using a 0-2 $M \mathrm{KCl}$ gradient in buffer consisting of $50 \mathrm{~m} M \mathrm{KH}_{2} \mathrm{PO}_{4}, 50 \mathrm{~m} M \mathrm{~K}_{2} \mathrm{HPO}_{4} \mathrm{pH} 6.5,1 \mathrm{~m} M$ DTT. Proteins were then frozen in liquid nitrogen and stored at $193 \mathrm{~K}$.

\subsection{Crystallization}

Spt16 NTD (13.3 $\mathrm{mg} \mathrm{ml}^{-1}$ ) was mixed with an equal volume of reservoir solution consisting of $0.1 \mathrm{M}$ sodium acetate $\mathrm{pH}$ 5.0, 17\%(w/v) PEG 3350, 0.1 $M$ ammonium iodide using the hanging-drop vapour-diffusion method at $292 \mathrm{~K}$. Crystals appeared after $1 \mathrm{~d}$ and were flash-cooled in $0.1 M$ sodium acetate $\mathrm{pH} 5.0,17 \%(w / v)$ PEG 3350, $0.1 M$ ammonium iodide, $20 \%(w / v)$ glycerol (Table 1$)$.
Table 3

Structure-solution and refinement statistics.

Values in parentheses are for the outer shell.

\begin{tabular}{ll}
\hline Resolution range $(\AA)$ & $61.64-1.84(1.88-1.84)$ \\
Completeness $(\%)$ & 100.0 \\
No. of reflections, working set & $53453(2634)$ \\
No. of reflections, test set & $2857(130)$ \\
Final $R_{\text {cryst }}$ & $0.156(0.203)$ \\
Final $R_{\text {free }}$ & $0.176(0.215)$ \\
No. of non-H atoms & \\
$\quad$ Protein & 3420 \\
$\quad$ Solvent & 458 \\
$\quad$ Total & 3878 \\
R.m.s. deviations & \\
$\quad$ Bonds $(\AA)$ & 0.008 \\
$\quad$ Angles $\left({ }^{\circ}\right)$ & 1.216 \\
Average $B$ factors $\left(\AA^{2}\right)$ & \\
$\quad$ Protein & 28.4 \\
Ramachandran plot & \\
$\quad$ Most favoured $(\%)$ & 98.9 \\
$\quad$ Allowed $(\%)$ & 100 \\
\hline
\end{tabular}

\subsection{Data collection and processing}

Diffraction data from human Spt16 NTD crystals were collected on beamline I02 at Diamond Light Source (DLS). Data obtained at $100 \mathrm{~K}$ were integrated, merged and scaled using the automated XDS (Kabsch, 2010; Winter, 2010) method applied at the beamline. Data-collection and processing statistics are shown in Table 2.

\subsection{Structure-solution and refinement statistics}

Human Spt16 NTD crystals belonged to space group F432 with one molecule in the asymmetric unit. Initial phases were

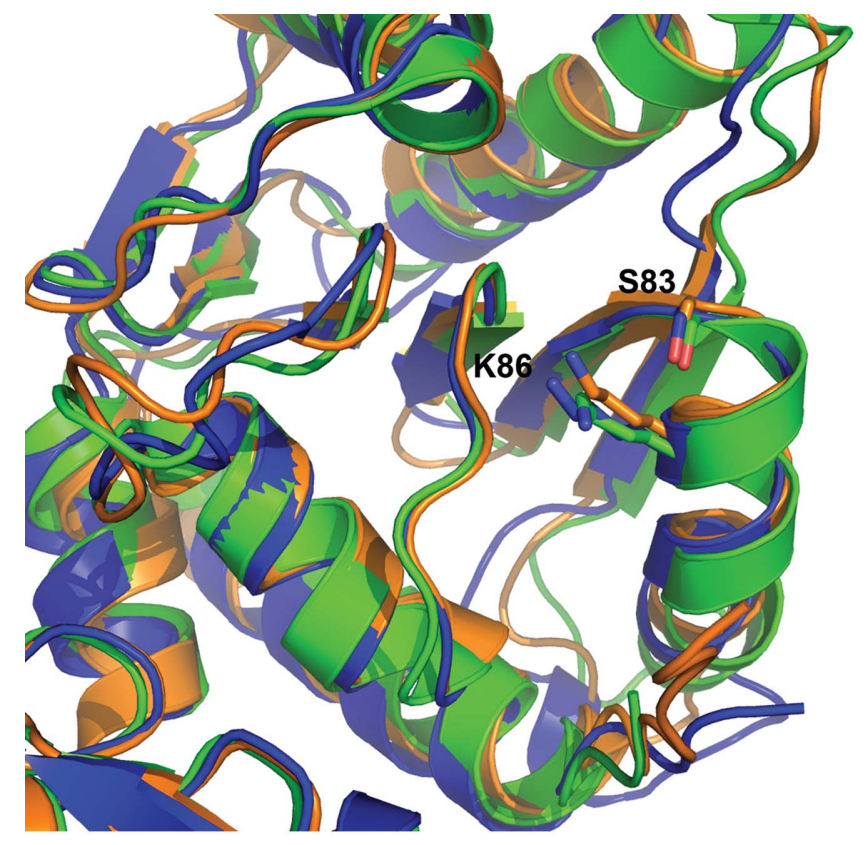

(a)

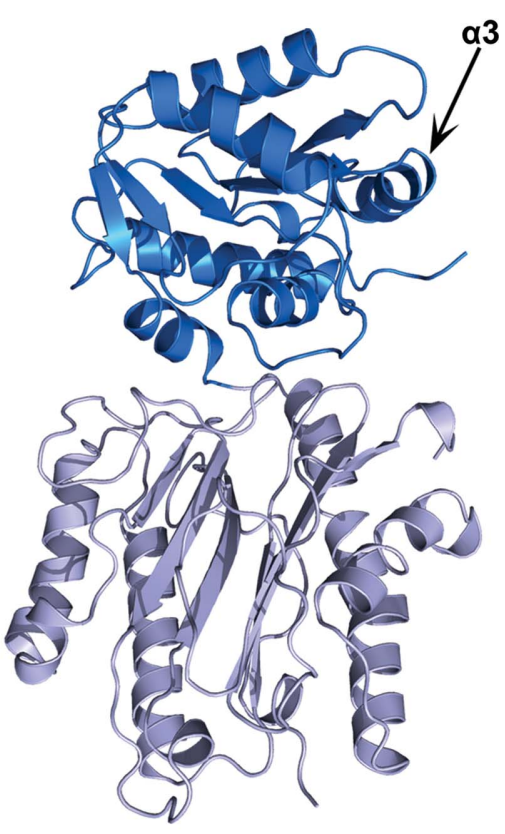

(b)

Figure 3

Putative binding site of Spt16 NTD for the H4 N-terminal tail. (a) Superimposition of Spt16 NTD structures from human (blue), S. cerevisiae (green; PDB entry 3bip) and S. pombe (orange; PDB entry 3cb5) showing a close-up view of the H4 N-terminal tail-binding site on the N-lobe. The Ser83 and Lys 86 side chains located on the $\alpha 3$ helix on the S. pombe Spt16 NTD structure and the corresponding Ser and Lys in human and S. cerevisiae Spt16 NTD are indicated. (b) Overall structure of human Spt16 NTD showing the location of the $\alpha 3$ helix. 
obtained by molecular replacement with Phaser (Storoni et al., 2004) using S. cerevisiae Spt16 NTD (PDB entry 3biq; 32\% sequence identity to human Spt16 NTD; VanDemark et al., 2008) as the initial search model. The model was built in Coot (Emsley \& Cowtan, 2004) and refined using PHENIX (Adams et al., 2010). The structure of human Spt16 NTD was refined to $1.84 \AA$ resolution. The model contains only chain $A$ (residues 2-432). Residues 433-510 were absent from the electron density. Side chains with poor electron density were built as stubbed residues. Details of the refinement statistics are shown in Table 3.

\subsection{Isothermal titration calorimetry}

Binding interactions between human Spt16 NTD and histones $\mathrm{H} 2 \mathrm{~A} / \mathrm{H} 2 \mathrm{~B}, \mathrm{H} 3 / \mathrm{H} 4$ or the $\mathrm{N}$-terminal tails of $\mathrm{H} 3$ or $\mathrm{H} 4$ were analysed by ITC using an iTC200 (MicroCal, GE Life Sciences). Experiments were conducted at $298 \mathrm{~K}$ with buffer

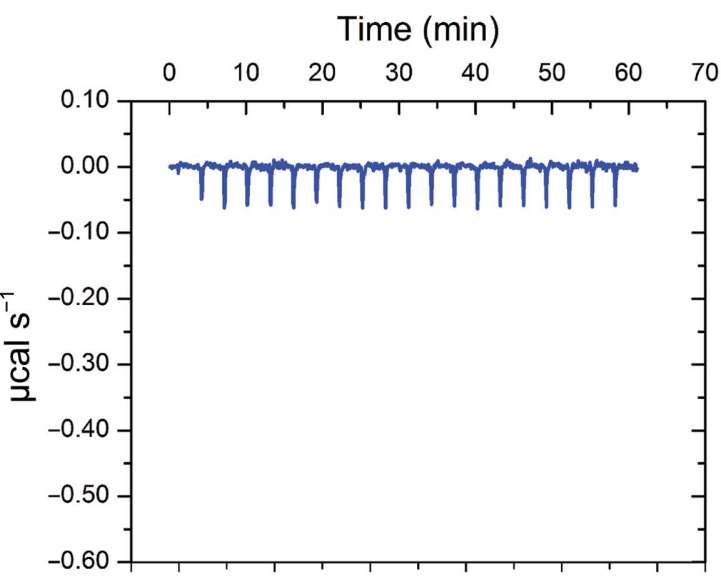

(a)

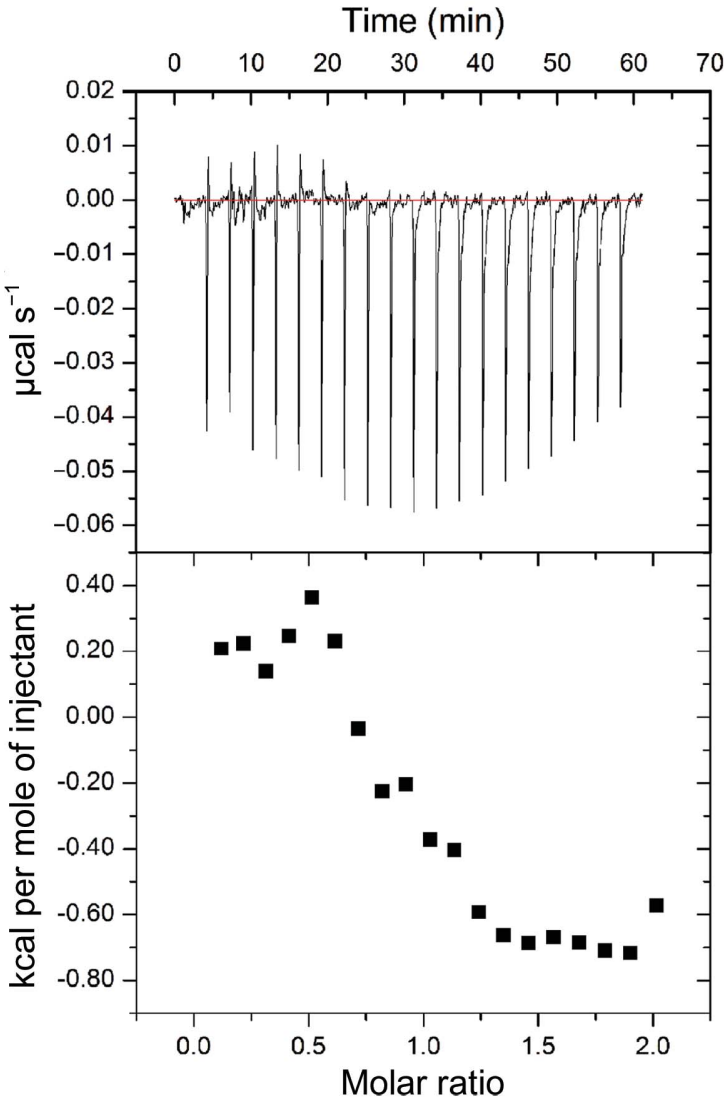

(c)

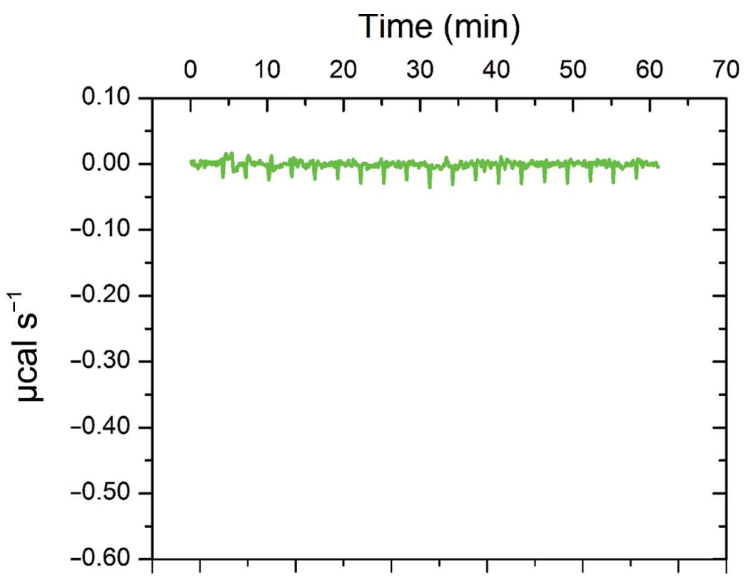

(b)

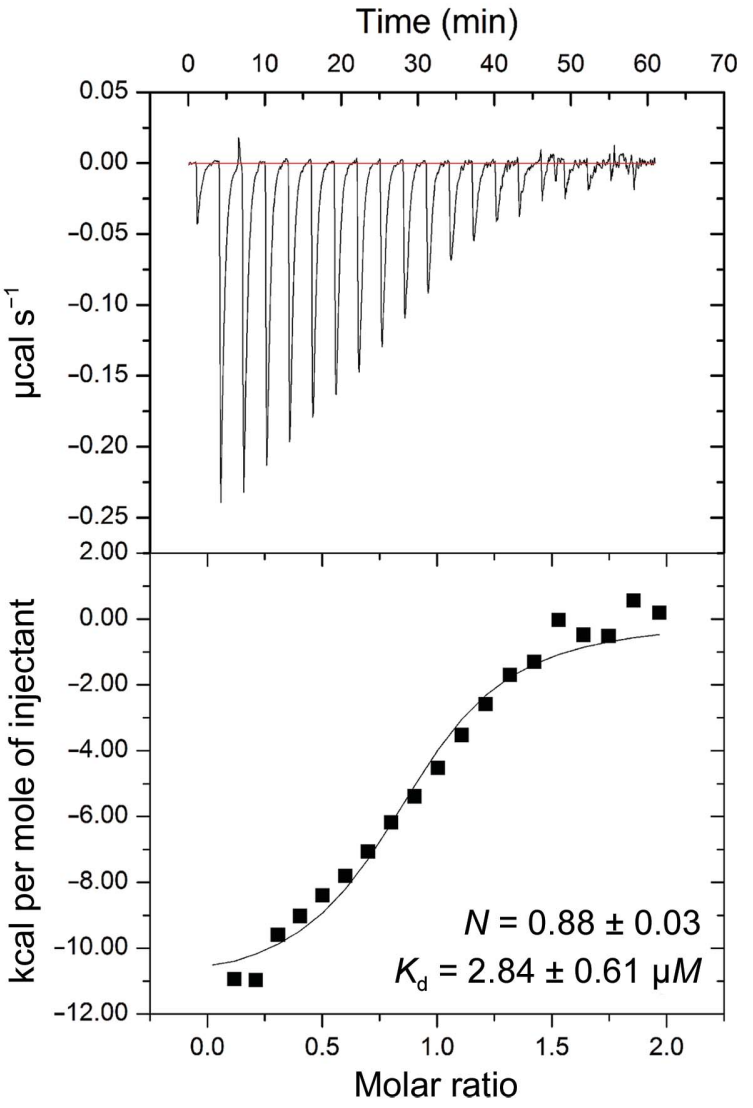

(d)

Figure 4

ITC profiles of human Spt16 NTD against (a) histone H4 N-terminal tail, (b) histone H3 N-terminal tail, $(c)$ H2A/H2B and $(d)$ H3/H4. $(a, b)$ Raw data for the N-terminal peptide from H4 (blue) and H3 (green), respectively, titrated into human Spt16 NTD. (c) ITC profile showing raw data (upper panel) and integrated raw data (lower panel) for the titration of human Spt16 NTD against H2A/H2B. (d) ITC profile showing raw data (upper panel) and integrated data (lower panel) for the titration of human Spt16 NTD against H3/H4. 
consisting of $20 \mathrm{~m} M$ HEPES-NaOH pH 7.5, $150 \mathrm{~m} M \mathrm{NaCl}$, $1 \mathrm{~m} M$ TCEP. Histone $\mathrm{H} 2 \mathrm{~A} / \mathrm{H} 2 \mathrm{~B}$ or $\mathrm{H} 3 / \mathrm{H} 4$ was loaded into the cell at a concentration of $30-40 \mu M$. Human Spt16 NTD was loaded into the injection syringe at a concentration ten times higher than that of the histones in the cell. For interactions of Spt16 NTD with H3 or H4 N-terminal peptides, human Spt16 NTD was loaded into the cell at a concentration of $50 \mu \mathrm{M}$ while histone $\mathrm{H} 3$ or $\mathrm{H} 4$ peptides were loaded into the syringe at a concentration 10-20 times higher than that of Spt16 NTD in the cell. 20 injections ( $2 \mu \mathrm{l}$ each) were added every $180 \mathrm{~s}$ to the cell. For control experiments, buffer was injected into the cell containing the protein. ITC data were generated by subtracting the raw data from the control experiment and were then analyzed using the Origin software (v.7). The histone N-terminal peptides sequences are MARTKQTARKSTGGKAPRKQLATKAARKSAPSTGGVKK for $\mathrm{H} 3$ (Abgent) and MSGRGKGGKGLGKGGAKRHRKVLRDN for $\mathrm{H} 4$ (Abgent). Peptides were weighed and dissolved in Milli-Q water at $50 \mathrm{mM}$ and then diluted into the appropriate buffer.

\section{Results and discussion}

\subsection{Crystal structure of human Spt16 NTD}

Our human Spt16 NTD construct consists of residues 1-510. The purified protein gave a band at between 49 and $62 \mathrm{kDa}$
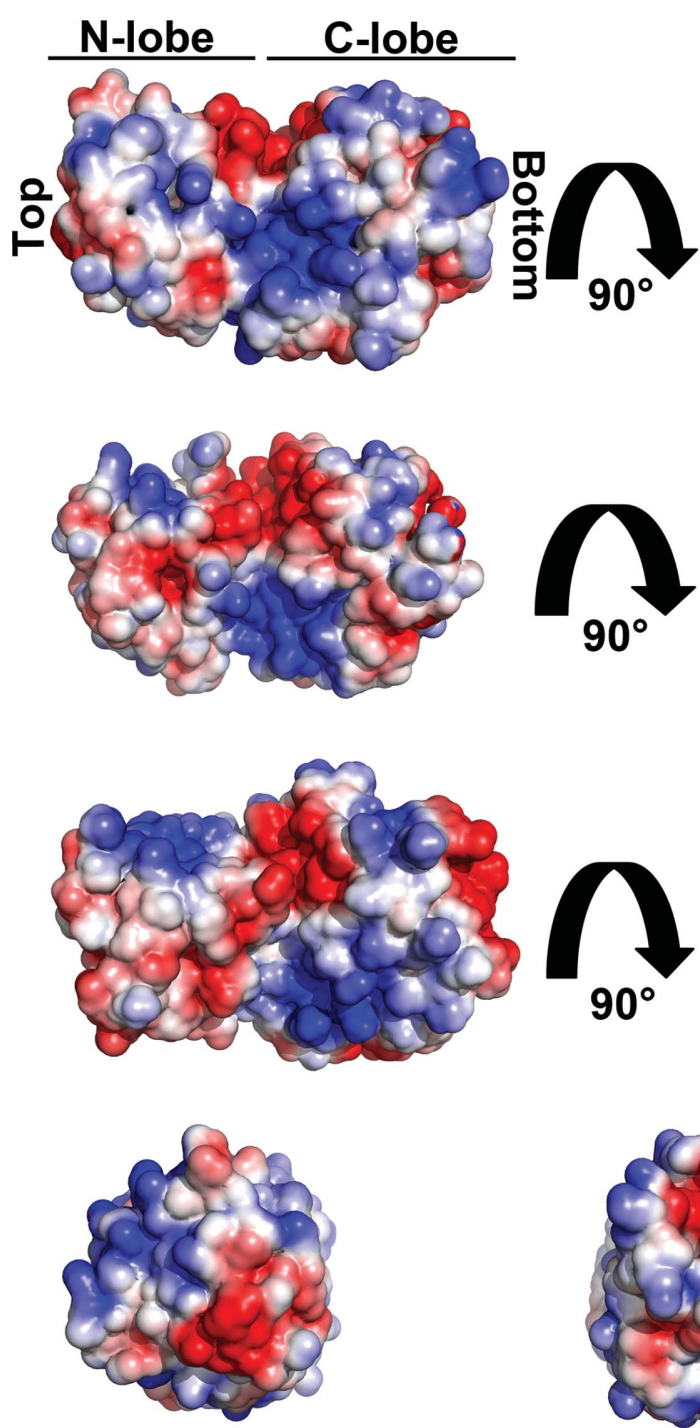

(d)

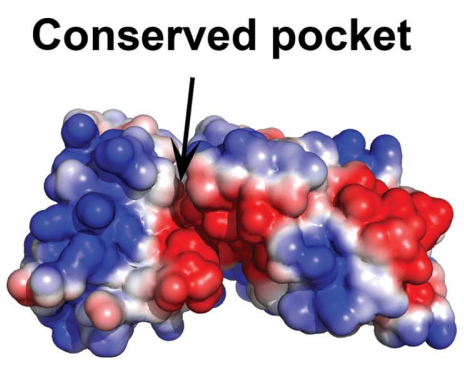

(a)
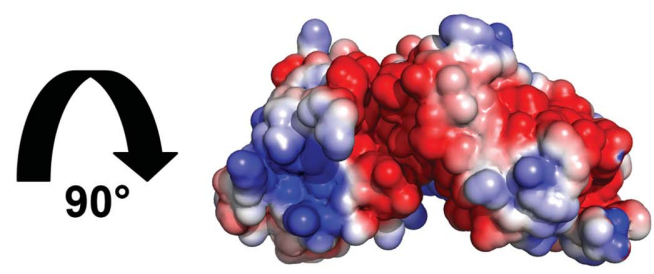

(b)
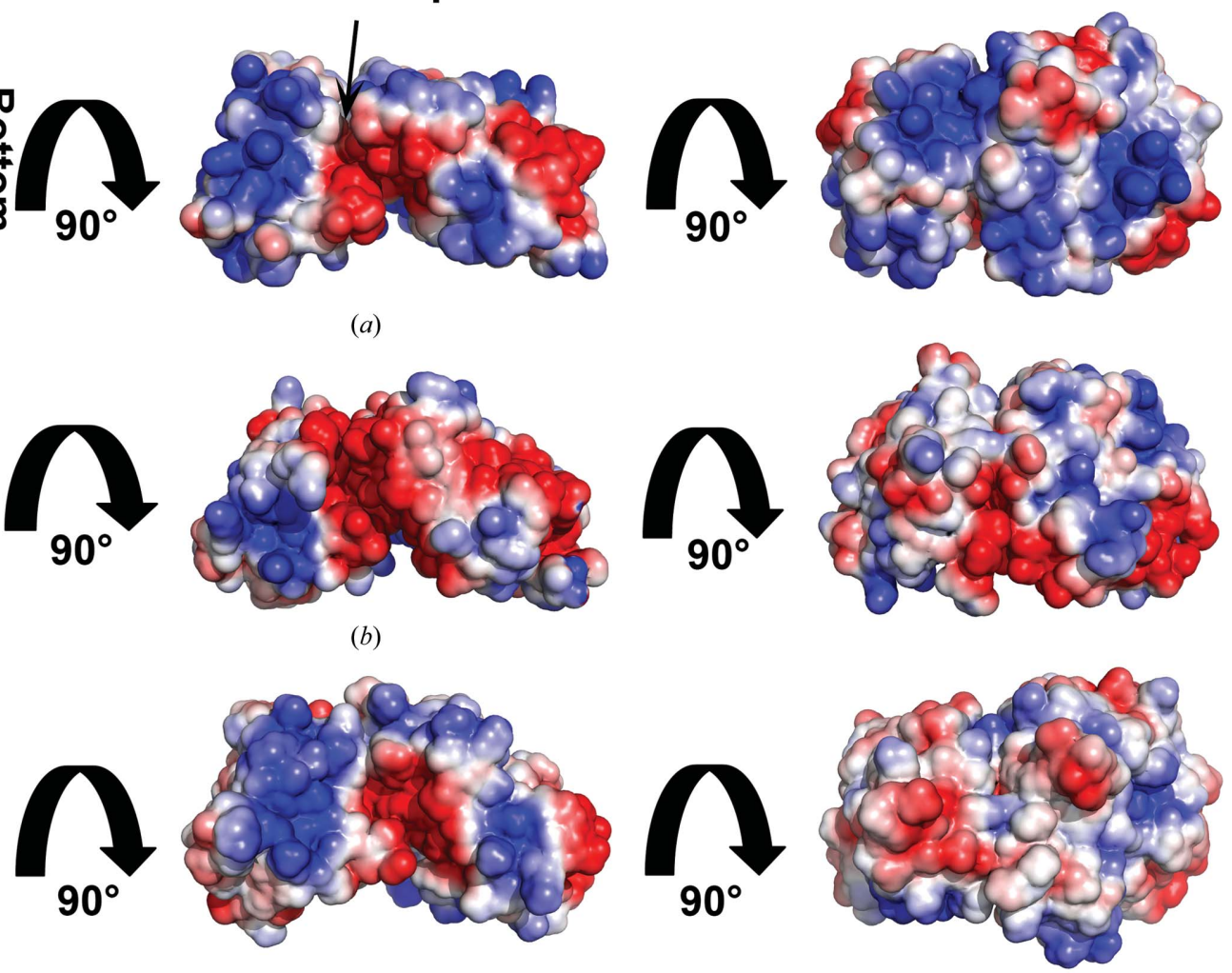

(c)

c)

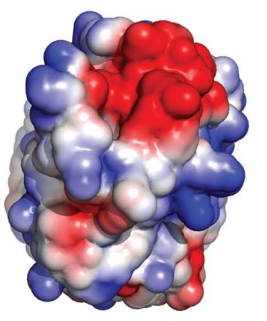

(e)

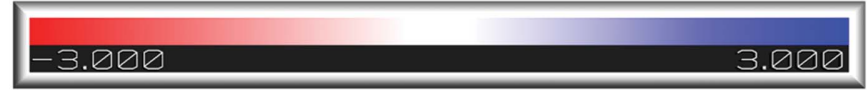

Figure 5

Electrostatic surface potential ( -3 to $3 k T)$ comparison of human, $S$. pombe and $S$. cerevisiae Spt16 NTD. Electrostatic surface representations of (a) human, (b) S. pombe and (c) S. cerevisiae Spt16 NTD in different views generated using APBS (Baker et al., 2001) in PyMOL (Schrödinger). S. pombe Spt16 NTD reveals a larger acidic surface at the conserved pocket than that observed in $S$. cerevisiae and human Spt16 NTD. The left panel is in the same orientation as in Fig. 2(a); the middle and right panels are rotated 90 and $180^{\circ}$ about the horizontal axis, respectively. ( $d, e$ ) Top and bottom view, respectively, of human Spt16 NTD as shown in the left panel in $(a)$. The arrow indicates the location of the conserved pocket. 
(Fig. 1a), which agrees with the calculated molecular weight of $57.9 \mathrm{kDa}$. The protein crystallized at $292 \mathrm{~K}$ after $1 \mathrm{~d}$ (Fig. $1 b$ ) and these crystals diffracted to a resolution of $1.84 \AA$ with space group F432 and one molecule of Spt16 NTD per asymmetric unit. While the construct contains residues 1-510, electron density for residues $433-510$ was not visible. The structure of human Spt16 NTD reveals two lobes: an N-lobe comprising residues $1-175$ and a C-lobe comprising residues 176-432. Although human Spt16 NTD shares only 32 and 35\% sequence identity with the structurally characterized $S$. cerevisiae and $S$. pombe Spt16 NTDs, respectively, it adopts a similar aminopeptidase-like fold in which the C-lobe resembles a pita-bread fold (Figs. $2 a$ and $2 b$; Stuwe et al., 2008; VanDemark et al., 2008). Superimposition of the human Spt16 NTD structure onto the structures of Spt16 NTD from S. cerevisiae (r.m.s.d. of $1.22 \AA$ for all $\mathrm{C}^{\alpha}$ atoms) and S. pombe (r.m.s.d. of $0.86 \AA$ for all $\mathrm{C}^{\alpha}$ atoms) shows slight differences in the loop regions in the $\mathrm{N}$ - and $\mathrm{C}$-lobes owing to variations in the amino-acid sequences and lengths (Figs. $2 c$ and $2 d$ ). Despite the presence of a linker between the two lobes, the orientation of the two lobes appears to be rigid across the three Spt16 NTD structures.

\subsection{Human Spt16 NTD binds histone H3/H4 with micromolar affinity}

Earlier studies on $S$. pombe Spt16 NTD show that it forms a 1:1 complex with the $\mathrm{H} 3 / \mathrm{H} 4$ dimer but that it does not bind the $\mathrm{H} 2 \mathrm{~A} / \mathrm{H} 2 \mathrm{~B}$ dimer in pull-down experiments. Moreover, it binds histone $\mathrm{H} 3$ and $\mathrm{H} 4 \mathrm{~N}$-terminal tails with low-micromolar affinity (Stuwe et al., 2008). A pocket harbouring Ser83 and Lys86 in $S$. pombe Spt16 NTD was shown to be involved in binding the $\mathrm{H} 4 \mathrm{~N}$-terminal tail. These residues are conserved in both human and $S$. cerevisiae Spt16 NTD and they adopt a similar configuration in all three structures (Fig. 3). This prompted us to investigate whether human Spt16 NTD has a role in binding histones and histone N-terminal tails.

To assess whether human Spt16 NTD binds the histone H3 and $\mathrm{H} 4 \mathrm{~N}$-terminal tails, we performed ITC experiments to measure the binding affinity between human Spt16 NTD and the $\mathrm{H} 3$ or $\mathrm{H} 4 \mathrm{~N}$-terminal peptides. ITC experiments showed that there was no enthalpy change when $\mathrm{H} 3$ or $\mathrm{H} 4$ peptide was added to human Spt16 NTD (Figs. $4 a$ and $4 b$ ), suggesting that human Spt16 NTD does not bind histone $\mathrm{H} 3$ or $\mathrm{H} 4 \mathrm{~N}$-terminal tails or that the interaction is too weak to be detected at the concentrations used in our assays. It is noteworthy that our assay was performed at $150 \mathrm{~m} M \mathrm{NaCl}$ concentration, while in the studies of $S$. pombe Spt16 NTD $25 \mathrm{~m} M \mathrm{NaCl}$ was used. Stuwe and coworkers have shown that the salt concentration influences the interaction of Spt16 NTD with histone tails in vitro (Stuwe et al., 2008). Unfortunately, human Spt16 NTD precipitated heavily and rapidly at $25 \mathrm{mM} \mathrm{NaCl}$, thus preventing further analysis. It is likely that these interactions depend on the ionic strength used in the in vitro binding assays and this may differ between species. Another possible explanation that may account for the differences in histone-tail binding abilities across different species is that the human and $S$. pombe Spt16 NTD structures have different electrostatic surface potentials (Fig. 5). Consistent with this suggestion, no binding of histone N-terminal tails is observed with $S$. cerevisiae Spt16 NTD (VanDemark et al., 2008).

To examine whether human Spt16 NTD binds histones, we performed ITC analyses of human Spt16 NTD against H2A/ $\mathrm{H} 2 \mathrm{~B}$ or $\mathrm{H} 3 / \mathrm{H} 4$. An ITC experiment with human Spt16 NTD against $\mathrm{H} 2 \mathrm{~A} / \mathrm{H} 2 \mathrm{~B}$ showed very little change in enthalpy when Spt16 NTD was added to $\mathrm{H} 2 \mathrm{~A} / \mathrm{H} 2 \mathrm{~B}$, suggesting that Spt16 NTD does not bind $\mathrm{H} 2 \mathrm{~A} / \mathrm{H} 2 \mathrm{~B}$ or binds with a very weak affinity. On the other hand, human Spt16 NTD binds histones $\mathrm{H} 3 / \mathrm{H} 4$ with low-micromolar affinity $\left(K_{\mathrm{d}}\right.$ of $2.84 \pm 0.61 \mu M$; $N=0.88 \pm 0.03$; Figs. $4 c$ and $4 d$ ). Together, these data show that human Spt16 NTD is likely to bind H3/H4 via the core histone.

\subsection{A conserved pocket on Spt16 NTD might contribute to} histone binding

To explore how human Spt16 NTD may interact with histones, we analysed the surface-residue conservation of Spt16 NTD to identify hypothetical patches that may be involved in histone binding. Sequence alignment of Spt16
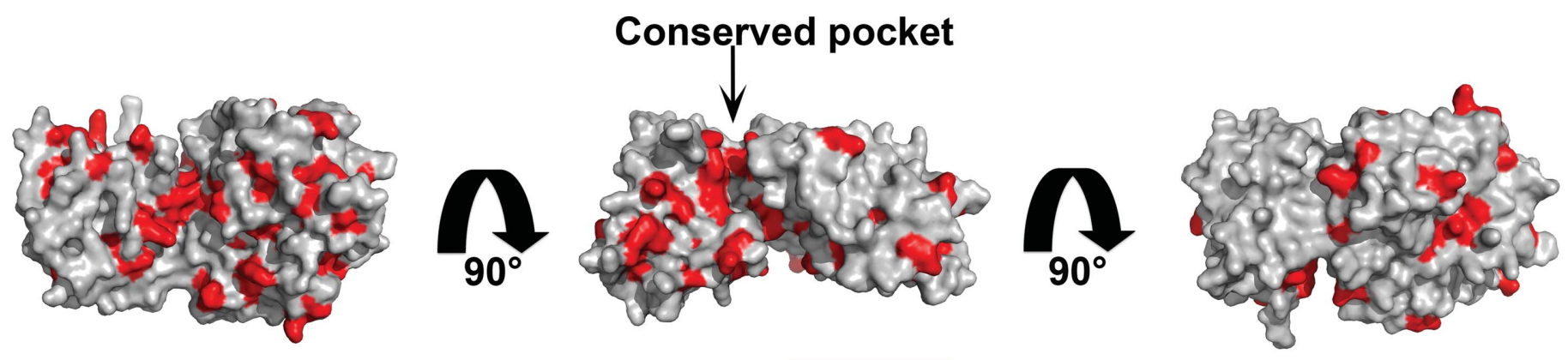

$0-80 \% \quad 80-100 \%$

Figure 6

Sequence conservation of Spt16 NTD. Sequence alignment of Spt16 NTD from human, Danio rerio, S. cerevisiae, S. pombe, Drosophila melanogaster, Xenopus laevis, Dictyostelium discoideum and Chaetomium thermophilum was performed and the sequence conservation was mapped onto the structure of human Spt16 NTD. $(a),(b)$ and $(c)$ are in the same orientation as in the left, middle and right panels of Fig. 5(a), respectively. The conserved pocket is indicated by an arrow. Residues that are more than $80 \%$ conserved are highlighted in red and the rest are coloured grey. 
NTD from various species was performed and the sequence conservation was mapped onto the structure of human Spt16 NTD (Fig. 6). The analysis revealed clusters of conserved residues on the $\mathrm{C}$-lobe and in a pocket between the $\mathrm{N}$-lobe and the C-lobe. Furthermore, electrostatic surface analysis of human Spt16 NTD showed an acidic patch that co-localizes within this conserved pocket (Fig. 5a, middle panel), suggesting a possible role of this region in histone interaction. The electrostatic maps also show acidic patches at the bottom of the C-lobe and at the top of the N-lobe that may be potential histone-binding sites (Figs. $5 d$ and $5 e$ ). Further studies are required to elucidate how Spt16 NTD interacts with histones. Our data are consistent with previous studies suggesting that Spt16 NTD is likely to play a supportive role with other FACT domains in modulating nucleosome assembly.

\section{Conclusion}

Little is known about Spt16 NTD and its functions. Here, we have reported the crystal structure of human Spt16 NTD and shown that it adopts a structure similar to those of S. cerevisiae and $S$. pombe Spt16 NTDs. Our ITC analyses showed that human Spt16 NTD is likely to bind histones H3/H4 through the histone core. Analysis of Spt16 NTD sequences from various species and the electrostatic surface of this domain reveal the presence of a conserved acidic pocket that may be involved in histone binding (Fig. 5a, middle panel, and Fig. $6 b$ ). Interestingly, some Spt16 NTD mutations that are lethal in conjunction with SSRP1 mutations cluster in a region near this conserved pocket (VanDemark et al., 2008). Further studies will be required in order to elucidate how FACT domains cooperate to bind histones.

\section{Acknowledgements}

We thank Gary Sibbet for helping us during histone purification and DLS for access to beamline I02 (mx8659), which contributed to the results presented here. This work was funded by Cancer Research UK. DTH was funded by ERC grant No. 647849.

\section{References}

Adams, P. D. et al. (2010). Acta Cryst. D66, 213-221.

Baker, N. A., Sept, D., Joseph, S., Holst, M. J. \& McCammon, J. A. (2001). Proc. Natl Acad. Sci. USA, 98, 10037-10041.
Belotserkovskaya, R., Oh, S., Bondarenko, V. A., Orphanides, G., Studitsky, V. M. \& Reinberg, D. (2003). Science, 301, 1090-1093.

Emsley, P. \& Cowtan, K. (2004). Acta Cryst. D60, 2126-2132.

Formosa, T., Eriksson, P., Wittmeyer, J., Ginn, J., Yu, Y. \& Stillman, D. J. (2001). EMBO J. 20, 3506-3517.

Gurard-Levin, Z. A., Quivy, J.-P. \& Almouzni, G. (2014). Annu. Rev. Biochem. 83, 487-517.

Hainer, S. J., Charsar, B. A., Cohen, S. B. \& Martens, J. A. (2012). G3, 2, 555-567.

Heo, K., Kim, H., Choi, S. H., Choi, J., Kim, K., Gu, J., Lieber, M. R., Yang, A. S. \& An, W. (2008). Mol. Cell, 30, 86-97.

Hondele, M., Stuwe, T., Hassler, M., Halbach, F., Bowman, A., Zhang, E. T., Nijmeijer, B., Kotthoff, C., Rybin, V., Amlacher, S., Hurt, E. \& Ladurner, A. G. (2013). Nature (London), 499, 111-114.

Kabsch, W. (2010). Acta Cryst. D66, 125-132.

Kasai, N., Tsunaka, Y., Ohki, I., Hirose, S., Morikawa, K. \& Tate, S. (2005). J. Biomol. NMR, 32, 83-88.

Kemble, D. J., McCullough, L. L., Whitby, F. G., Formosa, T. \& Hill, C. P. (2015). Mol. Cell, 60, 294-306.

Kemble, D. J., Whitby, F. G., Robinson, H., McCullough, L. L., Formosa, T. \& Hill, C. P. (2013). J. Biol. Chem. 288, 10188-10194.

Lambert, S. J., Nicholson, J. M., Chantalat, L., Reid, A. J., Donovan, M. J. \& Baldwin, J. P. (1999). Acta Cryst. D55, 1048-1051.

Luger, K., Mäder, A. W., Richmond, R. K., Sargent, D. F. \& Richmond, T. J. (1997). Nature (London), 389, 251-260.

Masse, J. E., Wong, B., Yen, Y.-M., Allain, F. H.-T., Johnson, R. C. \& Feigon, J. (2002). J. Mol. Biol. 323, 263-284.

McCullough, L., Rawlins, R., Olsen, A., Xin, H., Stillman, D. J. \& Formosa, T. (2011). Genetics, 188, 835-846.

Myers, C. N., Berner, G. B., Holthoff, J. H., Martinez-Fonts, K., Harper, J. A., Alford, S., Taylor, M. N. \& Duina, A. A. (2011). PLoS One, 6, e20847.

O'Donnell, A. F., Brewster, N. K., Kurniawan, J., Minard, L. V., Johnston, G. C. \& Singer, R. A. (2004). Nucleic Acids Res. 32, 58945906.

Orphanides, G., LeRoy, G., Chang, C.-H., Luse, D. S. \& Reinberg, D. (1998). Cell, 92, 105-116.

Orphanides, G., Wu, W.-H., Lane, W. S., Hampsey, M. \& Reinberg, D. (1999). Nature (London), 400, 284-288.

Storoni, L. C., McCoy, A. J. \& Read, R. J. (2004). Acta Cryst. D60, 432-438.

Stuwe, T., Hothorn, M., Lejeune, E., Rybin, V., Bortfeld, M., Scheffzek, K. \& Ladurner, A. G. (2008). Proc. Natl Acad. Sci. USA, 105, 8884-8889.

VanDemark, A. P., Blanksma, M., Ferris, E., Heroux, A., Hill, C. P. \& Formosa, T. (2006). Mol. Cell, 22, 363-374.

VanDemark, A. P., Xin, H., McCullough, L., Rawlins, R., Bentley, S., Heroux, A., Stillman, D. J., Hill, C. P. \& Formosa, T. (2008). J. Biol. Chem. 283, 5058-5068.

Winkler, D. D., Muthurajan, U. M., Hieb, A. R. \& Luger, K. (2011). J. Biol. Chem. 286, 41883-41892.

Winter, G. (2010). J. Appl. Cryst. 43, 186-190.

Xin, H., Takahata, S., Blanksma, M., McCullough, L., Stillman, D. J. \& Formosa, T. (2009). Mol. Cell, 35, 365-376.

Zunder, R. M., Antczak, A. J., Berger, J. M. \& Rine, J. (2012). Proc. Natl Acad. Sci. USA, 109, E144-E153. 